\title{
Pragmatic Function of the "Doge" Emoji on Weibo
}

\author{
Simin Xiong ${ }^{1, *}$ \\ ${ }^{I}$ Nanchang Business College of Jiangxi Agriculture University, Gongqing , Jiangxi,China \\ *Corresponding author. Email:Elise.xiong@outlook.com
}

\begin{abstract}
Emoji have become a pervasive communication tool through the proliferation of social media, it has several functions to facilitate the achievement of the communicative goal. Previous research concluded several functions of emoji. However, limited attention has been given to the function of a specific emoji. Thus, this research focuses on the pragmatic function of "Doge" emoji on Weibo. Based on earlier studies and Speech Act Theory, this study argues that "Doge" emoji has four functions, which are indicating humor, indicating irony, avoiding arguments and softening conflicts. To test this hypothesis, 104 Weibo users were invited to complete a questionnaire to judge the function of "Doge" emoji which is used on Weibo. Based on the analysis of the results, this research concluded that when used on "Weibo", Doge emoji has four functions. In addition, "Doge" emoji can express more than one function at the same time in one utterance. Third, the function of emoji connects closely with the context. It would transform with a change of context. Finally, it would cause misunderstandings when using "Doge" emoji since it could express several meanings.
\end{abstract}

Keywords: "Doge” emoji, Weibo, pragmatic function, Speech Act Theory

\section{INTRODUCTION}

Emoji have become a pervasive communication tool through the proliferation of social media. In contrast to face-to-face communication, we can not use gestures and facial expressions to facilitate the achievement of the communicative goal for both sides in virtual communication. To some extent, emoji is a supplement to online communication[7]. On Weibo, which is one of the most popular social media in China, the most ordinary emoji is ${ }^{\circ}$, called "doge" and it has rich meanings. This research aims to explore the pragmatic function of this emoji.

Previous studies have demonstrated that emoji are associated with informal interaction and they can build as well as maintain social bonds [6]. Yus [8] illustrates that there are 8 functions within the emoji, which has been the most complete summary so far. However, there are still some limitations to the previous studies. On the one hand, most of the studies focused on the one or two functions of the emoji [2], but few of them investigated one specific emoji deeply. On the other hand, the corpus of data they use is mainly from Twitter [1], Facebook, or WeChat[5], there has not been a study based on the corpus from Weibo. Thus, this study is to address the pragmatic function of "Doge" emoji on Weibo through tweets and users' comments. This research will based on the Speech Act Theory and analyze the locutionary, illocutionary and perlocutionary of the utterances with the use of "Doge" emoji.

The paper is structured as follows. Section 2 uses some specific examples to analyze the function of the doge emoji and its influence on the exact utterances. Section 3 analyzes the results of the questionnaire and concludes some limitations of "doge" emoji when using it. Section 4 concludes the paper with some open issues for future research.

\section{THE FUNCTIONS OF THE DOGE EMOJI ON WEIBO}

Based on the previous studies and speech act theory, this paper proposed that the functions of the doge emoji are (1) to indicate humor; (2) to indicate irony; (3) to avoid arguments; (4) to soften conflict.

\subsection{Indicating Humor}

When communicating on the social networks, paralinguistic cues are absent such as gestures or facial expressions[8]. It would cause misunderstanding or misinterpretation sometimes. Thus, it will contribute a 
lot to use emoji as a "contextualization cue" [3]. In this case, the users always add a "doge" at the end of the sentence as joking rather than serious. The following example taken from the tweets on Weibo can illustrate this function.

\section{A: Yizhi yong lengshui xilian hui zenmeyang?}

(what's the result of always washing your face with cold water?)

B:Ni de lian hui yizhi bei lengshui xi

(Your face will always be washed by cold water (2).)

From (1), A asks about the harm of washing your face with cold water seriously, B's answer does not really answer this question, but just repeats A's utterances in another way. The "Doge" emoji here is a signal of joking, A can understand that B's comment is joking rather than answering the question seriously with the use of this emoji. Assumed that B does not use the emoji, A will misunderstand that $\mathrm{B}$ is answering the question and may get annoyed.

(2) Japanese Ping-Pong player Niwa Kiko becomes famous in China because he does not seem to care about the result of the competition but is more eager to go to the concert.

A: Niwa laoshi qiaole jiemu qu kan Naimuban yanchnghui, hai kanle sanchang, ta kanshangqu zhen kaixin !

(Niwa didn't join the recording of the TV show, but went to the Naikuzaka's concerts three times, he looked

so happy!)

B: Yuanlai ta huixiao ye huitiao ${ }^{\circ}$ (Comment)

(So he can laugh and jump )

Since Niwa always behaves quiet and serious in the game, Weibo users always joke that he can not laugh. This tweet indicates that he looks happy when he goes to the concert, and the comment replies that "he can laugh and jump" just for humor. The comment does not mean he can laugh and jump literally. The illocutionary of this utterance is to express that Niwa is different in real life and create some punchlines at the same time. Adding a "Doge" emoji at the end of the sentence can be a marker of joking and contributes a lot to show his purpose.

\subsection{Indicating Irony}

Another important role of the doge emoji is to remind us that the utterances should not be understood literally. This sentence intends to express an ironic attitude. Consider the following example:

(3) A: \#Sun yingsha 3 bi 1 Ito Mima\# Wo shenzhi ganjue disanju shi rangqiu, fencha guoda.

(\#Yingsha Sun 3 to 1 Ito Mima\# I even feel that (Sun) conceded points in the third turn, because there is a huge score gap )

The context of this tweet is that Chinese player Sun beat the Japanese player Ito in the Ping-Pong games.

Because most of the Chinese audience do not like Ito due to her personality and style of playing Ping-Pong, lots of people post tweets or make some comments on Weibo as an irony. In this example, A does not really think Sun concedes her points during the games since the use of "doge"emoji . The illocutionary by adding this emoji is that A can easily show the purpose which is laughing at the skill of the Japanese player.

A: Zuifan zai jianyuli guanchiguanzhu, zhe shi shenme renjian tiantang !

( The criminal can eat and live in the prison without paying, What a heaven it is! )

This tweet alludes to the famous star Chris $\mathrm{Wu}$ who has just been sent to prison. By saying that, A does not mean that the prison is just like heaven literally, but expresses the ironic attitude that $\mathrm{Wu}$ was thrown in jail and will begin his prison life soon. Adding the Doge emoji could avoid misunderstandings and tell people that this is an irony.

\subsection{To Avoid Arguments}

When communicating on Weibo, there are always arguments or even verbal attacks. To avoid this, users would employ emoji "Doge" in the sentence. The illocutionary is to remind other users that the speakers do not focus on this issue seriously and implicate that they do not want to argue with anyone. Once other users understand their intention of using this emoji, the perlocutionary would be that they do not reply to the speakers.

A: Huiyuan guozhi zhen nanhe, goutou baoming 2

(Huiyuan juice tastes awful! Doge saves my life 
(I like sweet Tofu pudding.)

The context of (5) is that many famous brands including Huiyuan donate much during the flood disaster. So, people would argue intensely when others say something bad about these brands. In this example, $\mathrm{A}$ is complaining that Huiyuan juice tastes awful and worries that some impolitic netizens would have some arguments. Thus, A uses the emoji "Doge" at the end, the illocutionary of this emoji is to implicate that he does not want to argue with anyone. The perlocutionary of using emoji would be that the netizens understand A's intention and do not bother A or they still argue with A even if they know the meaning of this emoji.

(6) A: Shei zhidao xianzai zhan chulai weihu Liuxiang de ren dangnian youmeiyou zuiguo ta ne, goutou baoming



(Who knows whether those people standing for Xiang Liu now have ever accused him, doge save my life $e^{\bullet}$.)

The context of this utterance is that most people accused the athlete Xiang Liu of withdrawing from the Olympic Games several years ago. Recently, more and more people have stood up for him. In this example, A thinks it is ridiculous that some people hurt him before and now pretend they are just by standing up for him and attacking other people who disagree with them. By using "Doge"emoji , A intends to avoid an argument with those irrational people.

\subsection{Softening Conflict}

Sometimes we comment with opposite view when communicating on the social networks, but we don't mean to argue with someone. In this case, using doge emoji can contribute a lot to soften such a conflict.

A: Wo xihuan tian douhua.
B: Wo juede xian douhua geng haochi

(I think salty Tofu pudding tastes better (Comment)

In (7), A expresses his view about sweet Tofu pudding, but B disagrees with A. With the aid of emoji, their conflict can be softened. Provided that B does not use the emoji here, A would think that B comments on the opposite view intentionally and disputes with $\mathrm{B}$. The illocutionary of this emoji is to show B's purpose that he does not speak seriously and just wants to express his view. The perlocutionary would be that $\mathrm{A}$ does not consider it offensive when B disagrees with him.

A: Shaokao zhen haochi!

(Barbecue tastes great!)

B: Shaochi yidian, chi shaokao rongyi fapang

(Eating less barbecue, it would cause fat easily

.) $($ Comment $)$

In (8), A post a tweet that barbecue tastes great. But B suggests to A that eating barbecue would become fat easily. B's comment would disappoint A and arouse contradictions. To soften such conflicts, B uses the doge emoji at the end of the sentence to make his tone less serious.

\section{RESULT OF THE SURVEY}

To test the hypotheses, I invited 104 Weibo users who have ever used this emoji before to complete the questionnaires. The aim of this questionnaire is to judge the function of "Doge" emoji in different contexts. The results of this survey is presented in the tables below.

\subsection{Result of Indicating Humor}

Table 1 Function of indicating humor

\begin{tabular}{ccccccc}
\hline Context & $\begin{array}{c}\text { Indicate } \\
\text { humor }\end{array}$ & $\begin{array}{c}\text { Indicate } \\
\text { irony }\end{array}$ & $\begin{array}{c}\text { Avoid } \\
\text { arguments }\end{array}$ & $\begin{array}{c}\text { Soften } \\
\text { conflict }\end{array}$ & $\begin{array}{c}\text { Other } \\
\text { function }\end{array}$ & $\begin{array}{c}\text { Total } \\
\text { number }\end{array}$ \\
\hline & & & & & & \\
Example (1) & $95(91.35 \%)$ & $7(6.73 \%)$ & $2(1.92 \%)$ & $0(0 \%)$ & $0(0 \%)$ & $104(100 \%)$
\end{tabular}


Example (2) $75(72.12 \%) \quad 14(13.46 \%) \quad 9(8.65 \%) \quad 5(4.81 \%)$

$1(0.96 \%)$

$104(100 \%)$

From Table 1, in terms of Example 1, 91.35\% of people agree that in this context, "Doge" emoji is used to indicate humor. For the Example 2, 72.12\% of people consider that "Doge" emoji is supposed to indicate humor and $13.46 \%$ of people who suggest that the emoji is used to indicate irony in this example. Based on the data, most of subjects agree that "Doge" emoji is used to indicate humor or jokes in these two examples, which is in accordance with this paper's analysis.

\subsection{Result of Indicating Irony}

Table 2 Function of indicating irony

\begin{tabular}{ccccccc}
\hline Context & Indicate humor & $\begin{array}{c}\text { Indicate } \\
\text { irony }\end{array}$ & $\begin{array}{c}\text { Avoid } \\
\text { arguments }\end{array}$ & $\begin{array}{c}\text { Soften } \\
\text { conflict }\end{array}$ & $\begin{array}{c}\text { Other } \\
\text { function }\end{array}$ & $\begin{array}{c}\text { Total } \\
\text { number }\end{array}$ \\
\hline Example (3) & $30(28.85 \%)$ & $51(49.04 \%)$ & $18(17.31 \%)$ & $5(4.81 \%)$ & $0(0 \%)$ & $\begin{array}{c}104 \\
(100 \%)\end{array}$ \\
Example (4) & $6(5.77 \%)$ & $88(84.62 \%)$ & $6(5.77 \%)$ & $4(3.85 \%)$ & $0(0 \%)$ & 104 \\
& & & & & & \\
\end{tabular}

From Table 2, $28.85 \%$ of subjects consider that "Doge" emoji is used to indicate humor in Example 3 and $49.04 \%$ of subjects agree that the emoji is used to indicate irony. Based on the Table 1 and Table 2, it indicates that people would be confused about the function of indicating humor and indicating irony. According to Yus, the main difference between jokes and ironies is the explicit dissociative attitude they exhibit ([8]). If the boundary line is blurred between these two attitude, it would be difficult to divide from these functions. For example (2), some subjects consider that the emoji is used to express irony. Although it can be inferred that $\mathrm{B}$ has a positive attitude to Niwa since Niwa is popular in China, subjects do not recognize B's attitude from extent or they have a negative attitude to the Japanese athlete. Thus, they consider that the emoji is used to express an ironic attitude. For the result of example (3), it has a topic that \#Yingsha Sun 3 to 1 Ito
Mima\#, but does not have an explicit focus for the utterance. Besides, A is happy that Sun beat Ito. Thus, when the focus is Sun, this utterance can be seemed to express happy feelings and jokes for Sun. On the other hand, "doge" emoji can also become a signal of expressing ironic attitude to Ito when the focus is on her. For those who choose to avoid arguments, there are lots of Weibo users who stand up for Ito because they suggest to focus on the game rather than athletes. They always debate on the Internet. According to this background, some people would consider that they use this emoji to avoid arguments. For example (4), there is few disagreement because most of users have negative attitude towards $\mathrm{Chris} \mathrm{Wu}$, and express their ironic attitude on the Weibo.

\subsection{Result of Avoiding Arguments}

Table 3 Function of avoiding arguments

\begin{tabular}{ccccccc}
\hline Context & $\begin{array}{c}\text { Indicate } \\
\text { humor }\end{array}$ & $\begin{array}{c}\text { Indicate } \\
\text { irony }\end{array}$ & Avoid arguments & $\begin{array}{c}\text { Soften } \\
\text { conflict }\end{array}$ & $\begin{array}{c}\text { Other } \\
\text { function }\end{array}$ & $\begin{array}{c}\text { Total } \\
\text { number }\end{array}$ \\
\hline Example (5) & $28(26.92 \%)$ & $3(2.88 \%)$ & $71(68.27 \%)$ & $1(0.96 \%)$ & $1(0.96 \%)$ & $104(100 \%)$ \\
Example (6) & $6(5.77 \%)$ & $34(32.69 \%)$ & $48(46.15 \%)$ & $15(14.42 \%)$ & $1(0.96 \%)$ & $104(100 \%)$ \\
\hline $\begin{array}{c}\text { From Table 3, in terms of example (5), 68.27\% of } \\
\text { subjects agree that "Doge" emoji is used to avoid } \\
\text { arguments, 26.92\% of subjects consider that it is used to }\end{array}$ & $\begin{array}{c}\text { indicate humor. In (5), A said "Doge saves my life" at } \\
\text { the end of the sentence, it could be a signal of avoiding } \\
\text { verbal ack. For example (6), there are some }\end{array}$
\end{tabular}


disagreements, $32.69 \%$ of subjects consider that the emoji is used to indicate irony and $14.42 \%$ of people suggests that it is used to soften conflict. For the view of indicating irony, it could be inferred from the context that A have an ironic attitude to the netizens who blame Liu before but attack others online to stand up for Liu. The "doge" emoji become a signal of irony. However, A said "Doge saves my life" at the end of the utterance, it implicated that he does not want anyone to debate with him. Thus, this emoji is more like a signal to avoid arguments. For softening conflicts, the presupposition of this function is that there exists disagreement or conflict. However, in (6), A post this tweets with no comments. Thus, there is no conflict in this example.

\subsection{Result of Softening Conflicts}

Table 4 Function of softening conflicts

\begin{tabular}{ccccccc}
\hline Context & $\begin{array}{c}\text { Indicate } \\
\text { humor }\end{array}$ & $\begin{array}{c}\text { Indicate } \\
\text { irony }\end{array}$ & $\begin{array}{c}\text { Avoid } \\
\text { arguments }\end{array}$ & $\begin{array}{c}\text { Soften } \\
\text { conflict }\end{array}$ & $\begin{array}{c}\text { Other } \\
\text { function }\end{array}$ & $\begin{array}{c}\text { Total } \\
\text { number }\end{array}$ \\
\hline Example (7) & $6(5.77 \%)$ & $0(0 \%)$ & $20(19.23 \%)$ & $78(75 \%)$ & $0(0 \%)$ & $104(100 \%)$ \\
& & & & & & \\
Example $(8)$ & $25(24.04 \%)$ & $0(0 \%)$ & $23(22.12 \%)$ & $55(52.88 \%)$ & $1(0.96 \%)$ & $104(100 \%)$
\end{tabular}

From Table 4, 75\% of subjects agree that this emoji is used to soften conflict. $19.23 \%$ of subjects consider that it is used to avoid arguments. In (7), there are conflicts between A and B. With usage of the emoji, it illustrates that B does not intend to strengthen disagreement but to express his point of view. For the function of avoiding arguments, it always happens when there is potential possibility to receive some malicious comments and would cause a bitter quarrel. In (7), the topic does not concerning anything sensitive. Thus, there is no potential to receive malicious comments. For example (8), there are some disagreements, $24.04 \%$ of subjects consider that the emoji is used to indicate humor and $22.12 \%$ of subjects argue that it was used to avoid arguments. In (8), the emoji could be used to indicate humor when A and B are close friends and they are talking about last party that they joined together. Under this context, $\mathrm{B}$ is joking with $\mathrm{A}$. When there is no evidence indicating the relationship between $\mathrm{A}$ and $\mathrm{B}$, it can be inferred from the utterance that B does not want A eat too much barbecue and there are some conflicts. To soften such contradicts, B uses emoji to make his utterances less serious. Based on the analyze, it can be concluded that the function of emoji would transform with change of context[4]. Besides, it would cause misunderstandings when using the "Doge" emoji since it could express several meanings.

\section{CONCLUSION}

This paper analyzes the pragmatic function of the popular emoji "Doge" on Weibo. Major conclusions drawn from the present study are the followings. First, "Doge" emoji has four functions, which is indicating humor, indicating irony, avoiding arguments, softening conflicts. Second, "Doge" emoji can express more than one function at the same time in one utterance. Third, the function of emoji connect closely with the context. It would transform with change of context. Finally, it would cause misunderstanding when using "Doge" emoji since it could express several meanings.

For future research, the focus will be the conclusion of the context which influence the function of "Doge" emotion. Besides, in this paper, only 104 people did the questionnaire. More subjects will be invited in future study. In addition, this study focus on the function of "Doge" emoji on Weibo, future research would explore the function this emoji on other social media such as Wechat.

\section{REFERENCES}

[1] Corina, Parkwell. Emoji as social semiotic resources for meaning-making in discourse: Mapping the functions of the toilet emoji in Cher's tweets about Donald Trump - Science Direct[J]. Discourse, Context \& Media, 30:100307-100307.

[2] Dresner E , Herring SC . Emoticons and Illocutionary Force[J]. Springer Netherlands, 2012. Huang G H, Chang CT, Bilgihan A, et al. Helpful or harmful? A double-edged sword of emoticons in online review helpfulness[J]. Tourism Management, 2020, 81(1):104135.

[3] Gumperz J J . Discourse strategies: Socio-cultural knowledge in conversational inference. 1982.

[4]Hsieh S H , Tseng T H . The effects of emoticons and text-messaging on social interaction: Playfulness in mobile instant messaging [J]. 2015.

[5]Li L, Yue Y. Pragmatic functions of emoji in internet-based communication---a corpus-based study[J].Asian-Pacific Journal of Second and Foreign Language Education, 2018, 3(1):16.

[6] Mcculloch G. Marcel Danesi. 2017. The semiotics of emoji: The rise of visual language in the age of the 
internet. London: Bloomsbury Academic. Pp. 208.

[J]. Canadian Journal of Linguistics, 2019, 65(1):1-5.

[7] Will G, Huang P, Yu Q . Emoji and communicative action: The semiotics, sequence and gestural actions of 'face covering hand'[J].Discourse Context \& Media, 2018:S2211695818300801-.

[8] Yus F. Not all emoticons are created equal[J]. Linguagem Em Curso, 2014, 14(3):511-529. 